Candido Portinari, Construirão casas, 1956

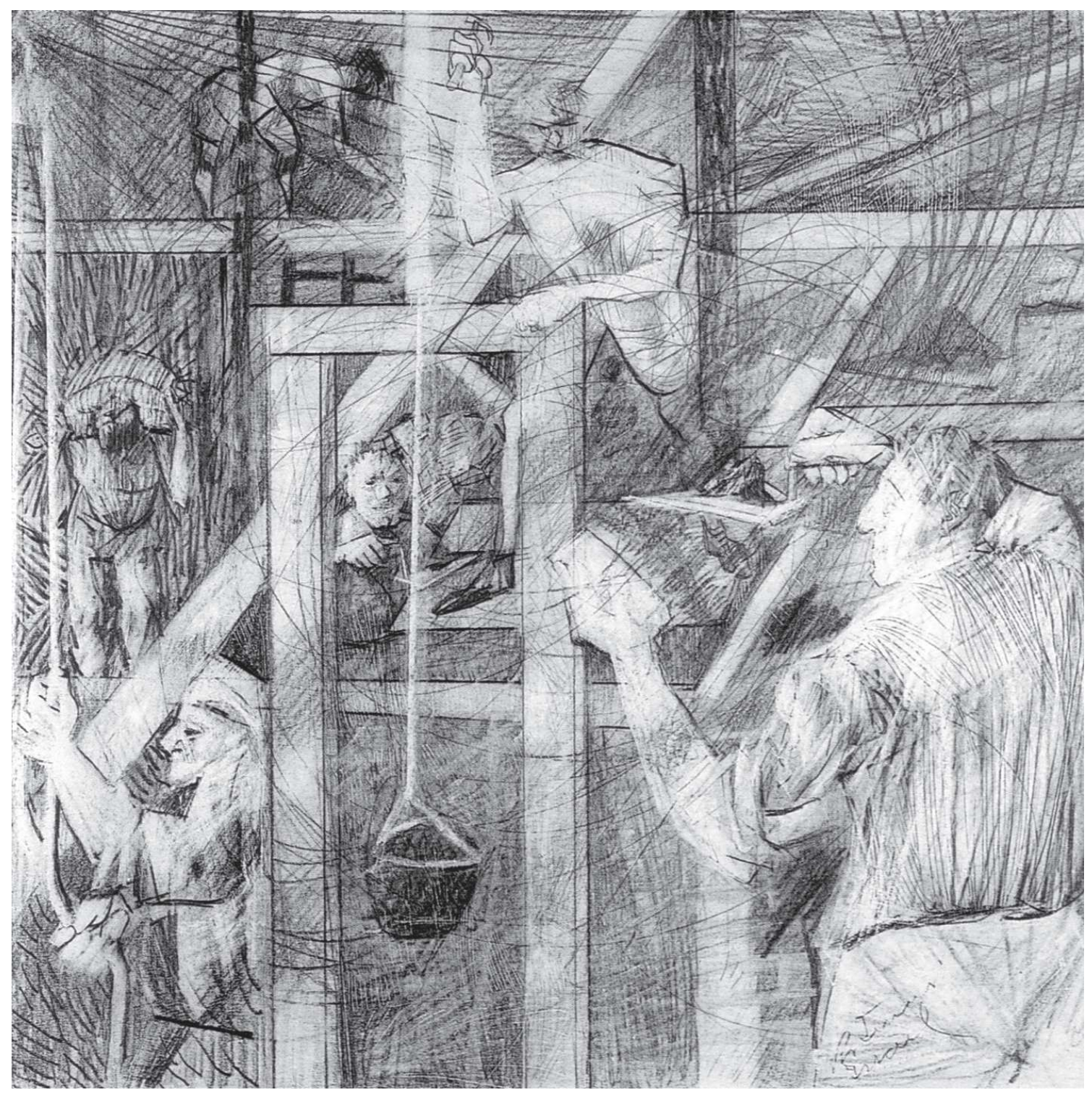




\title{
La Importancia de las Ideias en el Cambio de Paradigma: Aplicación a las Políticas de Servicios Sociales y Asistencia Social en España (1982-2008)
}

\author{
José Adelantado* \\ Roser Pérez-Giménez**
}

\begin{abstract}
Resumen: Este trabajo muestra la existencia de un cambio de paradigma en las políticas de Servicios Sociales y Asistencia Social en España. El análisis se realiza mediante el método de marcos interpretativos. Se estudia el cambio en las ideas y los discursos que contienen las leyes de esas políticas, y se concluye que dicho cambio procede de la conjugación de factores endógenos (estructura institucional doméstica) y exógenos (europeización), que habrían provocado la emergencia de nuevos actores (sub y supra nacionales, institucionales y extrainstitucionales, públicos y privados), portadores de nuevos intereses, ideas y discursos.

Palabras clave: Cambio de paradigma; marcos interpretativos; intereses; ideas y discursos; servicios sociales; asistencia social; europeización.
\end{abstract}

Keywords: Paradigm change; frame analysis; interest; ideas and discourses; personal social services; social assistance; europeization.

\footnotetext{
* Licenciado en Ciencias Económicas y doctor en Sociología; profesor titular del Departamento de Sociología de la UAB. Ha sido investigador principal en varios proyectos con financiación pública. Su área de interés son las relaciones entre la estructura social y las políticas sociales. Es coordinador del libro Cambios en el Estado de Bienestar: políticas sociales y desigualdades en España; ha publicado varios artículos en revistas especializadas y de divulgación (Papers, Revista Internacional de Sociología, Revista Mexicana de Sociología, Journal of European Social Policy, Mientrastanto, Barcelona Societat, Carrer); y es autor de una veintena de capítulos en libros sobre políticas de bienestar en sectores como pensiones, vivienda, inmigración, rentas mínimas de inserción, servicios sociales, o planes de inclusión. Universidad Autónoma de Barcelona, José Adelantado Gimeno, Facultad de Ciencias Políticas y de Sociología, Campus UAB - Edificio B, 08193 - Cerdanyola (Barcelona), España.

** licenciada en Sociologia y Ciencias Políticas y doctora en Sociologia por la Universidad Autónoma de Barcelona, Diplomada en Enfermería y Trabajo Social por la Universidad de Barcelona; Coordinadora de titulación de enfermería y profesora de Sociologia en la EU Cruz Roja-UAB de Terrassa. Ha participado como investigadora en varios proyectos en el Seminario de Análisis de Politica Social SAPS-UAB en el área de Desigualdades sociales en Salud y Política Sanitaria, donde colabora en el seguimiento y tutorización de tesinas y tesis dentro del campo de la salud; ha publicado en revistas especializadas y de divulgación (Salut 2000-España, Katalysis-Brasil; Medicina social-UAM-Mèxico); participó en el Informe Foessa sobre vulnerabilidad social y exclusión social y en la formación continuada dirigida a políticos y cuadros técnicos municipales de los Planes de Inserción en Cataluña. Universidad Autónoma de Barcelona, José Adelantado Gimeno, Facultad de Ciencias Políticas y de Sociología, Campus UAB - Edificio B, 08193 - Cerdanyola (Barcelona), España.
} 


\section{Introducción}

El objetivo de este escrito es analizar las continuidades y cambios en las políticas autonómicas de servicios sociales, rentas mínimas de inserción y planes autonómicos de inclusión social entre 1982 y 2008. En el trabajo se conectan tres campos de discusión académica sobre la reforma de los sistemas de bienestar: los cambios de paradigma de las políticas, su europeización y el poder causal de las ideas y discursos públicos.

¿Cómo se ha producido el cambio institucional en la política de Servicios Sociales y Asistencia Social en España? ¿Hacia dónde se han dirigido las reformas? ¿Qué discursos utilizan los gestores políticos? ¿Cómo ha reaccionado España a la influencia de la Unión Europea? ¿Se pueden establecer diferencias entre Comunidades Autónomas en la aplicación de los discursos?. La hipótesis a contrastar es que desde la transición democrática hasta la actualidad, se ha producido un cambio de paradigma institucional en las políticas autonómicas de SS y AS. Dicho cambio se ha producido por acumulación de reformas sobre continuidades históricas, a través de sucesivas adaptaciones a los requerimientos de la europeización.

La transición a principios de la década de 1980's de las políticas keynesianas a monetaristas en el Reino Unido estudiada por (Hall 1993), abrió una interesante discusión sobre los cambios de paradigma de políticas. Según este autor, un paradigma de política es un armazón interpretativo de ideas y patrones, que especifica no sólo los objetivos de política y el tipo de instrumentos que pueden usarse para conseguirlos, sino también la naturaleza de los problemas y la forma de pensarlos y comunicarlos. A su vez, esta discusión ha alimentado el debate teórico sobre el teorema de los patrones de dependencia, tanto al señalar las limitaciones de la idea del cambio como reproducción endógena, como al abrir nuevas perspectivas con la idea del cambio institucional como secuencias reactivas que no tienden a reproducir los patrones sino a transformarlos (Mahoney 2000; Ebbinghaus 2005).

El concepto de europeización, si se entiende como gobernaza multinivel puede ser un recurso para comprender el nexo que afecta a la recomposición vertical y horizontal del poder doméstico. De hecho la europeización opera simultáneamente hacia arriba bottom-up y hacia abajo top-down (Börzel 2002); y puede ser útil para entender cómo se han reducido las resistencias para la reforma institucional en los estados de bienestar mediante un cambio discursivo. En el estudio se parte de la perspectiva de Radaelli (2003) que identifica cuatro reacciones diferentes de los estados ante los cambios inducidos por la Unión Europea. Especialmente interesa la reacción de tipo "absorción" que entiende el cambio como adaptación, y la reacción de "transformación" que equivale a una modificación sustancial de los instrumentos y de los objetivos de las políticas.

El estudio empírico del cambio de paradigma se realiza a través de la selección de algunos "marcos interpretativos" en las leyes de servicios sociales, rentas mínimas de inserción y planes autonómicos de inclusión social. El enmarcado framing es una metodología de investigación frame analysis según la cual un marco es el conjunto de creencias y significados orientados hacia la acción, que legitima las actividades de un grupo (Snow y Benford, 1992). El análisis de marcos parte de considerar las condiciones de producción y difusión de elementos ideológicos y culturales así 
\} LA IMPORTANCIA DE LAS IDEIAS EN EL CAMBIO DE PARADIGMA: (...) - ADELANTADO, J. e PÉREZ-GIMÉNEZ, R. \}

como las condiciones sociales para su viabilidad o fracaso. Los marcos son un andamio conceptual, un esquema interpretativo tanto de las causas como de las soluciones a un problema. Los marcos seleccionan, codifican y condensan una parte de la realidad. Los seis marcos interpretativos que se han utilizado, se analizan desde lo que Schmidt (2008) Ilama "institucionalismo discursivo", que pretende captar el poder explicativo de las ideas y los discursos, en la medida que un cambio institucional puede emerger tras un cambio cognitivo o normativo en los valores o las creencias de los actores.

Los resultados apuntan que ante un contexto de austeridad permanente que venía recomendando una contención de costes, las políticas autonómicas de SS y AS se han expandido -posiblemente como compensación a la contracción de las políticas contributivas- y liberalizado por medio de: (a) la introducción de mecanismos a favor del mercado y del Tercer Sector (voluntariado social), (b) la introducción de mecanismos que refuerzan la responsabilidad individual y la subsidiariedad del Estado, (c) la exigencia de predisposiciones, conductas y contrapartidas para merecer las ayudas. Además, la competencia exclusiva de estas políticas por parte de las Comunidades Autónomas (CCAA) muestra d) un efecto mímesis (isomorfismo, replicación normativa) y dominó (breve secuencia temporal), y e) una convergencia discursiva en su formulación junto a una gran heterogeneidad en su implementación.

\section{Patrones de dependencia, europeización y poder causal de la ideas en los cambios de paradigma}

Una conceptualización muy elemental del teorema de los patrones de dependencia ("path dependence") se basa en el argumento de que los acontecimientos anteriores afectan a los posteriores. En la literatura sobre el institucionalismo se distinguen por lo menos dos interpretaciones del "path dependence" (Mahoney, 2000): una en relación a la idea de reproducción, basada en secuencias de inercia y autorefuerzo donde el cambio se entiende como una pérdida del equilibrio y su recuperación; y otra, basada en la idea de secuencias reactivas donde el cambio se entiende como una cadena de acontecimientos temporalmente ordenados y causalmente conectados, que no tienden a reproducir los patrones sino a transformarlos, a través de una cadena de reacciones y contrareacciones. En el primer caso se trata de un sistema cerrado con un mecanismo de retroalimentación interno, cuya única vía para el cambio es un factor exógeno; así el modelo determinista sólo puede explicar los casos estables de path dependence. En cambio, en el segundo caso, desde una perspectiva más dinámica, Ebbinghaus (2005) entiende que el path dependence restringe la elección pero no determina el paso adaptativo siguiente, y considera tres escenarios para la transformación institucional (a) adaptación marginal al entorno sin cambio en los principios path stabilization (b) adaptación gradual con renovación parcial de las instituciones y redirección de los principios path departure (c) acontecimiento que acaba con el autorefuerzo en una institución establecida que puede dar paso a otra nueva en su lugar path cessation o path switching.

Entre las explicaciones del cambio institucional a partir del teorema del path dependence para explorar los cambios de paradigma, la teorización más reconocida 
es la de Hall (1993). Según este autor, un paradigma de política policy paradigm es un armazón interpretativo de ideas y patrones, que especifica no sólo los objetivos de política y el tipo de instrumentos que pueden usarse para conseguirlos, sino también la naturaleza de los problemas y la forma de pensarlos y comunicarlos (discursos). Hall estudia cómo se pasa de un paradigma de política a otro a partir de tres órdenes de cambio. Primero, ajuste de los instrumentos disponibles. Segundo, cambio de instrumentos o técnicas (basado en el aprendizaje de la experiencia) pero sin modificar la jerarquía de objetivos. Tercero, cambio radical y simultáneo de instrumentos y objetivos (cambio del discurso político).

El teorema del path dependence, como los distintos institucionalismos, son muy útiles para comprender las realidades de las instituciones a escala nacional-estatal (como el paradigma keynesiano en economía), pero muestran limitaciones explicativas al no tomar en cuenta factores "exógenos" como son los procesos de globalización o europeización. Según Schmidt (2008), tampoco parece que las explicaciones que provienen tanto del teorema del path dependence como la tradición teórica de los "viejos" institucionalismos, han considerado seriamente el papel de las ideas en los procesos de cambio. Como mucho, en ambos casos las ideas se han considerado más como un factor que contribuye a la estabilidad que al cambio. Frente a esa visión estática del papel de las ideas, el "institucionalismo discursivo" que propone Schmidt entiende que el cambio institucional puede emerger tras un cambio cognitivo o normativo en los valores o las creencias subjetivas de los actores. En este sentido, la transformación institucional se explica por la inconsistencia de los marcos cognitivos dominantes en la sociedad y la deslegitimación de la institución. La emergencia de nuevas ideas pone en duda el esquema cognitivo y puede llevar a un cambio de paradigma. Además, los "viejos" institucionalismos entienden que las normas y preferencias que tienen los actores son fijas. En cambio, desde el institucionalismo discursivo, los intereses y las normas son cambiantes ya que responden a intereses de los actores.

Börzel (2002) propone resumir las grandes conceptualizaciones sobre la relación entre la Unión Europea y sus estados miembros en dos procesos; bottom-up en el que se institucionaliza el sistema político Unión Europea mediante la creación y consolidación de instituciones supranacionales, y top-down cuando penetran reglas y normas europeas en esferas domésticas anteriormente diferenciadas. Una de las definiciones más articuladas y utilizadas, del concepto de europeización se debe a Radaelli (2003). Para él, la europeización incluye: procesos de construcción, difusión e institucionalización de reglas formales e informales; procedimientos; paradigmas de política; estilos o formas de hacer las cosas; valores y normas compartidas que inicialmente se definen y consolidan en el proceso político de la UE, para ser incorporados posteriormente en la lógica del discurso; las estructuras políticas y las políticas públicas internas (estatales y subestatales). Radaelli también identifica cuatro reacciones diferentes de los estados ante los cambios inducidos por la UE. La primera es la "inercia" que consiste en una ausencia de cambio; tiene lugar cuando la adaptación es demasiado costosa para generar una reforma; y se puede manifestar mediante resistencias y retrasos en la adaptación de normas y directivas. La segunda reacción es la "absorción" que indica cambio como adaptación; en este caso las 
\} LA IMPORTANCIA DE LAS IDEIAS EN EL CAMBIO DE PARADIGMA: (...) - ADELANTADO, J. e PÉREZ-GIMÉNEZ, R. \}

estructuras domésticas y el legado político se acomodan a los requerimientos y pueden absorber cambios no fundamentales mediante una combinación de resistencia y flexibilidad, pero manteniendo el núcleo de objetivos y el entramado esencial de la política. La tercera es la "transformación" que es similar a lo que Hall (1993) Ilama "tercer orden" de cambio, es decir, un cambio de paradigma. Y la cuarta reacción ante los cambios inducidos por la UE, paradójicamente, puede ser una "reducción" de europeización, en cuyo caso la política nacional sería menos europea que antes.

Las reacciones de inercia y absorción, en parte, se pueden equiparar con los dos primeros órdenes de cambio que identifica Hall. El primer nivel corresponde a un cambio en la configuración de los instrumentos mientras el segundo nivel corresponde a un cambio de instrumentos. En los dos casos, los objetivos de las políticas públicas no se modifican. Así mismo, corresponden a los escenarios path stabilization y path departure que destaca Ebbinghaus. Una adaptación marginal de la institución responde a cambios en el entorno mientras una adaptación gradual con una renovación parcial, o una redirección limitada, de los principios claves se da cuando los cambios en el entorno son significantes y los recursos para la adaptación suficientes. Los cambios son graduales, se acumulan a lo largo del tiempo y pueden dar lugar finalmente a reorientaciones importantes. La gran mayoría de los cambios se dan al nivel secundario porque las creencias son fácilmente ajustables en función de las nuevas informaciones y de las experiencias pasadas. Por contra, el tercer nivel de cambio identificado por Hall correspondería al tercer resultado posible que describe Radaelli como "transformación" de las políticas públicas en cuanto se modificarían los objetivos de las mismas, o al path switching señalado por Ebbinghaus.

Una parte de los trabajos sobre europeización se han dedicado al estudio del cambio doméstico. Bulmer y Radaelli (2003) describen un mecanismo de cambio en el proceso de europeización que tiene que ver con los paradigmas que produce la UE y distinguen entre la integración positiva y la negativa. Es interesante el caso de la integración positiva, que tiene lugar cuando la UE prescribe la adopción de un modelo determinado en ciertas áreas de políticas. En este caso, los estados miembros deben adecuarse al modelo supranacional. Esta "presión para la adaptación" pasa por los mecanismos de coerción y de mimetismo. Entonces la difusión de las políticas públicas europeas ejerce una presión normativa sobre los estados miembros para sustituir sus paradigmas por el paradigma definido por la UE. Este mecanismo opera cuando la UE utiliza instrumentos hard de políticas públicas a través de reglamentos y directivas. En cambio, existen otros mecanismos soft en cuanto no se basan en instrumentos legislativos o una coerción directa de Bruselas. Incluso muchas veces no existe presión por adaptación sino todo lo contrario, los estados utilizan los cambios europeos como excusa para incentivar cambios domésticos que ya estaban impulsando.

Otro de los aspectos más debatidos sobre la europeización es el cambio que la misma produce como consecuencia de la transferencia de soberanías hacia arriba y la ejecución de legislación hacia abajo. Según Hooghe y Marks (2001), la fragmentación del estado en múltiples unidades subnacionales, junto con la integración de los estados en organizaciones intergubernamentales o supranacionales, provoca 
un reparto vertical del poder entre múltiples niveles de gobierno, y un reparto horizontal entre múltiples actores. El estado es desafiado desde arriba, desde abajo y desde los costados (Loughlin, 2007); desde arriba, el estado es desafiado por la integración y la globalización. Desde los costados, el estado está siendo erosionado por el nuevo papel de la sociedad civil en el siglo XXI. Desde abajo, el estado está experimentando una gran movilidad subnacional. En este sentido, la emergencia de un "nuevo regionalismo" tiene su origen en una pérdida de centralidad del estado ante los desafíos de la globalización y el surgimiento de nuevos espacios de gobierno y gobernanza, tanto a nivel nacional como supranacional y subnacional.

\section{Discusión general}

Un cambio de paradigma de política implica la sustitución de un armazón interpretativo de la naturaleza de los problemas, los objetivos de la política y los instrumentos para resolverlos, por otro distinto. Si se comparan las ideas que orientaban los grandes objetivos de las primeras leyes autonómicas de SS, así como los instrumentos y técnicas a aplicar en aquel contexto, con el armazón contemporáneo, se puede hablar de un cambio de paradigma. En efecto, los principios de universalismo y responsabilidad pública proclamados en las leyes, típicos de un Estado del Bienestar Keynesiano, se han sustituido por selectivismo (grupos de riesgo) y responsabilidad individual, entre otros; típicos de un Estado del Bienestar Schumpeteriano (Jessop, 2007). Sin embargo, la transición de un paradigma a otro no se ha realizado por ruptura, sino por reformas acumulativas que descartan las interpretaciones reproductivas del teorema del path dependence. Así, aún entendiendo que la historia condiciona, pero que, a su vez, no determina el paso reactivo siguiente, cabe acogerse a las interpretaciones del cambio estructural en la política por acumulación y cambios en el contexto. Se trata de una adaptación gradual con renovación parcial de las instituciones y redirección de los principios, que corresponde al escenario path departure de Ebbinghaus (2005) y se puede equiparar al segundo orden de cambio propuesto por Hall (1993).

El desarrollo de los SS y AS no se puede entender exclusivamente por razones endógenas; es decir, por requerimientos de la política doméstica, sino que es preciso tener en cuenta la visión de España como problema y de Europa como solución que dominó en buena medida el discurso políticos durante diversos lustros después de la muerte de Franco, alrededor del triángulo democratización-modernización-europeización (Powell, 2007). Ciertamente, los procesos que señala Börzel top-down y bottom-up son clave para entender el cambio institucional, y la emergencia de una estructura de poder multinivel (Hooghe y Marks, 2001), que implica un reparto vertical entre múltiples niveles de gobierno, y un reparto horizontal del poder entre múltiples actores.

Si bien las primeras leyes de SS se inspiraron en valores típicos de los estados del bienestar keynesianos (especialmente los del régimen corporativo-conservador); desde principios de los 90 en España la política de SS inicia un proceso de reforma en el que progresivamente la legislación incorporará, primero nuevos instrumentos y después nuevos objetivos; en ambos casos a partir de una reacción del Estado español a los cambios inducidos por la Unión Europea, primero de "absorción" y 
\} LA IMPORTANCIA DE LAS IDEIAS EN EL CAMBIO DE PARADIGMA: (...) - ADELANTADO, J. e PÉREZ-GIMÉNEZ, R. \}

después de "transformación", todo ello a través de un proceso "top-down" y en el marco de una gobernanza multinivel. El cambio de instrumentos (segundo orden de cambio de Hall, y escenario path departure de Ebbinghaus) tiene que ver con el abandono del objetivo político del monopolio público en la prestación de servicios, y se ilustra en el reconocimiento jurídico de las diversas formas de privatización mercantil, así como del recurso al voluntariado social. A ese objetivo también responde la puesta en marcha de "la última malla de seguridad" (safety net), los programas de RMI. Con estos programas se inicia una expansión institucional de los SS tradicionales hacia la AS (gestión de transferencias de rentas no contributivas), y se suma a un cambio de objetivo más amplio de "recalibración" de las políticas "contributivas-pasivas" hacia las "asistenciales-activas".

El concepto de "europeización" de Radaelli es útil para explicar el proceso arriba-abajo señalado por Börzel, como la penetración de normas y reglas europeas en la lógica del discurso, las estructuras políticas y las políticas públicas internas (estatales y subestatales). La europeización de la política de SS y AS en España se ha realizado mediante un proceso de persuasión y aprendizaje social de estructuras cognitivas y normativas, a través de mecanismos "soft" al no basarse en instrumentos legislativos, como reglamentos y directivas o una coerción directa de Bruselas (a diferencia por ejemplo, de las sanciones que conlleva el incumplimiento de los criterios de convergencia monetaria establecidos en Maastricht que opera con mecanismos "hard"). La incorporación de marcos interpretativos como la gravedad de la pobreza, el derecho a la inserción o la cohesión social, proceden de presiones europeas. En términos de Radaelli, la reacción del Estado español a las presiones procedentes de la UE ha sido primero de "absorción", mediante una combinación de resistencia y flexibilidad, que estaría marcada por la reforma de las primeras leyes de SS y la puesta en marcha de las RMI, y después una reacción de "transformación", un progresivo cambio de objetivos desde la lucha contra la pobreza hacia la inclusión social y la "activación". Esto, según Castel (2002), significa una gran cambio para esta política, que iría desde la atención tradicional a los marginados por parte de los SS, a la gestión del no-trabajo.

La gobernaza multinivel como característica de la europeización, es un tanto posterior al proceso constituyente en lo relativo a la descentralización autonómica. Durante la transición la idea de una España integrada en Europa, sobre todo desde la perspectiva de los nacionalismos vasco y catalán, suponía una cesión de "competencias hacia abajo", a las CCAA, y a la vez "hacia arriba", a Bruselas, lo que haría disminuir notablemente el peso del Estado. Parecía que una combinación de "más Europa" y "menos España" podría contribuir a resolver (o conllevar) el problema nacional/estatal español (Quintanilla, 2001; citado por Powel, 2007: 55). El proceso arriba-abajo "top-down" es relevante para comprender el cambio de paradigma en los SS y la AS y su relación con una estructura de poder multinivel. Desde la instauración del Método Abierto de Coordinación en la Cumbre de Lisboa en marzo del 2000, que viene a reforzar el viejo principio de subsidiariedad, la política social queda en manos de los estados miembros; mientras que la Comisión Europea queda reducida a un papel de estímulo, coordinación y potenciación de aprendizaje mutuo, pero con escasos mecanismos de coerción y sanción. El instrumento que mejor lo 
ilustra son los Planes Nacionales de Acción para la Inclusión social (PNAin), y sobre todo su implementación a escala autonómica y local. En ellos se observa que un reparto vertical de los niveles de gobierno puede conducir a una cierta desresponsabilización europea, estatal y regional, transferida a los municipios; mientras que un reparto horizontal del poder, posibilita una ventana de oportunidades políticas tanto de actores mercantiles como de la iniciativa social. Pero además, la gobernanza multinivel afecta tanto a distintos actores institucionales (consejerías, ayuntamientos, diputaciones) como a otros agentes sociales (organizaciones empresariales, sindicales, de afectados, de voluntarios...).

Para Radaelli, las estructuras normativas y cognitivas incluyen los valores, las normas y los discursos. Por una parte, la transformación cognitiva puede cambiar las preferencias y el comportamiento de los decisores políticos. De hecho, el proceso de europeización tiene que ver con discursos, mapas cognitivos, marcos normativos y estilos que constituyen el contexto en el cual los actores intervienen. A su vez Schmidt sostiene que el cambio institucional puede ocurrir tras un cambio cognitivo o normativo en los valores o las creencias de los actores, así la emergencia de nuevas ideas pone en duda el marco cognitivo previo y puede llevar a un cambio de paradigma. Ahora bien, para que un factor actúe por medio de un discurso, es preciso un estado de cosas, determinadas condiciones, que posibiliten la acción causal de dicho factor, las ideas. Según estos autores, las ideas serían el factor causal del cambio institucional, mientras que los discursos serían el mecanismo por medio del cual se produce el cambio.

\section{Ideas y discursos en las leyes autonómicas de SS}

En este apartado se presenta una secuencia de algunos marcos interpretativos en las leyes autonómicas de servicios sociales (SS) desde la transición democrática hasta el año 2008. Se procede mediante el análisis longitudinal de tres marcos interpretativos que van desde el universalismo genérico de las primeras leyes, hasta un discurso reciente sobre los derechos subjetivos, pasando por un período intermedio (desde principios de los noventa a principios de los dos mil) en que emerge la ideología neoliberal (anexos).

Marco interpretativo 1: Universalismo y responsabilidad pública (1982-92)

Entre 1982 y 1992, todos los gobiernos autonómicos desarrollan sus primeras leyes de SS, amparados en la competencia "exclusiva" que permite el art. 148.1. $20^{\circ}$ de la Constitución Española. Los ejes comunes en el discurso de estas primeras leyes es la "estatalización" de los SS a través de las ideas-eje de universalismo y responsabilidad pública. El principio de igualdad y universalidad implica que los SS deben ir dirigidos a todos los ciudadanos con el reconocimiento de que todos ellos tienen derecho al bienestar social. La finalidad de la aplicación de este principio de universalidad es evitar la estigmatización a la que eran sometidos determinados grupos de población a los que se dirigían los servicios asistenciales. El universalismo se proponía como la contestación teórica e ideológica a la beneficencia y el asistencialismo que caracterizaba la época franquista (Roldán y García, 2006). El principio de responsabilidad pública implica que las administraciones públicas deben 
\} LA IMPORTANCIA DE LAS IDEIAS EN EL CAMBIO DE PARADIGMA: (...) - ADELANTADO, J. e PÉREZ-GIMÉNEZ, R. \}

comprometerse con la prestación de SS, facilitando y dotando a la comunidad de los recursos financieros, técnicos e institucionales necesarios. La responsabilidad y financiación pública se concebía como la solución a un sistema benéfico y residual en el que tenían preeminencia las organizaciones altruistas de asistencia tradicional (Cáritas, Cruz Roja, ONCE); esto es, "un sistema público estricto con disciplina dura para las entidades voluntarias y excluyente de las mercantiles" (Casado, 2007: 136).

El derecho a los servicios sociales no aparece reflejado en la Constitución ni en los Estatutos de Autonomía de forma explícita. La ley de Bases del Régimen Local (7/1985) tampoco clarifica el reconocimiento y la responsabilidad de los servicios sociales; si bien estipula que la prestación de servicios sociales y de reinserción social es competencia municipal, y obligatoria para los municipios de más de 2000 habitantes, no concreta en qué consisten estos servicios. Así, las primeras leyes de SS no garantizan el derecho, tan sólo enuncian aspiraciones generales. Carecen del mecanismo para reclamar y hacer efectivo el ejercicio del derecho, de previsión y compromiso para dotar a los sistemas de recursos económicos y humanos. La descentralización contenida en la Constitución de 1978 y desplegada por los Estatutos de Autonomía, afectó a la organización institucional para el desarrollo de esta política y ha contribuido a conformar en realidad un mosaico de 17 sistemas con grandes dificultades para una coordinación formal entre CA y Estado, y con desigualdades territoriales inter e intraautonómicas.

\section{Marco interpretativo 2: Privatización y altruismo (1993-2003)}

Al año siguiente de finalizar el período de promulgación de las primeras leyes, se inicia por parte de Galicia en 1993, un proceso de reforma hacia un modelo más liberal a través de cambios incrementales que se extiende hasta la actualidad. No hay acuerdo entre los especialistas sobre si son segundas leyes o leyes de segunda. El auge, y progresiva consolidación de la ideología neoliberal en el gobierno de la economía (un cambio de paradigma del keynesiano al monetarista, según Hall, 1993) también tuvo lugar en la reorientación de los servicios sociales a través de los "marcos" discursivos de privatización y voluntariado social (ONG's).

Las segundas leyes, de forma irregular pero progresiva introducen cambios que tienden hacia la liberalización y privatización de los SS en las CCAA. Se va produciendo un reconocimiento expreso de la presencia de la iniciativa privada lucrativa e integración de la misma en el sistema, así como el reconocimiento del voluntariado social (entre 1991 que empezó Cataluña y 2004 que acabó Murcia, las 17 CCAA aprobaron leyes de voluntariado social) organizado alrededor del Ilamado Tercer Sector. Con la iniciativa mercantil se establece un sistema de conciertos (también llamado externalización) para la prestación de servicios (asistencia domiciliaria, residencias para la tercera edad...). En algunas CA se transfieren recursos monetarios directamente a los usuarios mediante la figura del cheque-servicio, y gana terreno la idea la participación de los usuarios en la financiación del coste de los servicios. 


\section{Marco interpretativo 3: Subjetivación de derechos y copago (2003-08)}

Las nuevas leyes de SS, especialmente las que se reforman a partir del 2000, propiamente una segunda generación (La Rioja, 2002; Asturias, 2003; Madrid, 2003; Murcia, 2003; Cataluña, 2006; Navarra, 2006; Cantabria, 2007, País Vasco, 2008, Galicia, 2008) empiezan a esbozar un discurso alrededor del "marco" de los derechos subjetivos. Se empieza a concretar el derecho mediante la garantía de las prestaciones a detallar en las Ilamadas Carteras de Servicios. Así mismo se está afianzando la garantía jurídica del acceso universal especialmente a las prestaciones técnicas o básicas. En algunos casos como en Cataluña (2006), se ha individualizado el derecho, de modo que en la comprobación de medios sólo se tiene en cuenta los recursos de la persona, no de la unidad familiar. Simultáneamente, también se está explicitando en estas leyes un conjunto de derechos y deberes de los usuarios, ausentes en la primera generación. Se puede destacar entre las innovaciones (Alemán y García, 2005: 50-51) el derecho a una información suficiente y veraz sobre las prestaciones y recursos, a participar en la toma de decisiones que les afecten, y a elegir libremente el tipo de medidas y recursos a aplicar. Y entre las obligaciones, se incluyen: destinar las prestaciones recibidas para el fin que se concedieron; comprometerse a participar activamente en el proceso de mejora, autonomía e inserción social; acudir a las entrevistas con los profesionales; y realizar las actividades indicadas del proceso de integración social. Por otra parte, se refuerza la idea de la participación de los usuarios en la financiación de los servicios, no mediante impuestos, sino a través de un copago individualizado, para cuya regulación se establecerá un módulo que aportará la administración y un complemento a cargo del usuario, para lo cual se tendrá en cuenta sus ingresos y su patrimonio. Algunas de las leyes anticipan un cambio de instrumentos para fortalecer una tendencia hacia las transferencias monetarias, antes que al desarrollo de los servicios. La ley valenciana (1997) ya se refiere al Bono-Residencia para la tercera edad y discapacitados, y la ley madrileña (2003) al chequeservicio a personas y familias.

\section{Las ideas y los discursos de la UE sobre la pobreza, la inserción y la inclusión. La reacción de España}

En este apartado se realiza un análisis del discurso sobre la pobreza y la exclusión social de la UE y la reacción de España. Se estudia la secuencia de creencias y significados de los programas de lucha contra la pobreza desde la segunda mitad de los ochenta, hasta el discurso contemporáneo sobre la activación laboral. Se considera la sucesión de tres marcos interpretativos que ilustran la emergencia, consolidación y revisión del paradigma neoliberal; presencia retórica de la pobreza y exclusión (hasta mediados del los noventa), complementariedad de objetivos crecimiento-empleo-cohesión (durante la década siguiente), y reorientación del discurso desde ideas proteccionistas a productivistas mediante el slogan de la activación, más recientemente.

En España el discurso sobre la pobreza entra en la agenda política y se institucionaliza como "última malla de seguridad", a través de las rentas mínimas de inserción (RMI) desde los gobiernos autonómicos (1989-1995). Progresivamente el marco "lucha contra la pobreza" se vincula al marco de la "inclusión social" desde 
\} LA IMPORTANCIA DE LAS IDEIAS EN EL CAMBIO DE PARADIGMA: (...) - ADELANTADO, J. e PÉREZ-GIMÉNEZ, R. \}

principios del año 2000, con la presentación de los planes nacionales de acción para la inclusión social (PNAIn) y su réplica en las CA (PAI). Progresivamente desde el 2000 las políticas de protección social giran alrededor de las políticas de empleo; el "marco de la inclusión" se reinterpreta como "activación", es decir, hacer que trabajar sea rentable (making work pay).

\section{Marco interpretativo 4: Pobreza y derecho a la inserción (1975-1996)}

En el campo de la pobreza la UE ha llevado a cabo tres Programas-marco de Acción Comunitaria entre 1975 y 1994. De 1975 a 1979 Pobreza (I), desde 1979 hasta 1984 no hubo acciones; entre 1985-89 Pobreza (II); de 1989 a 1994 Pobreza (III), en este programa se abandona la idea de los colectivos específicos y se pone el acento en tres dimensiones: la pluridimensionalidad de la pobreza, la participación de los menos favorecidos y el partenariado (participación de agentes sociales, políticos y económicos). En el curso del mismo se transita del concepto de pobreza hacia el de exclusión social. De forma simultánea a Pobreza (III) se produce una intensa actividad en torno a las rentas mínimas. El Parlamento Europeo recomendó en 1988 el establecimiento de rentas mínimas garantizadas en todos los países de la UE. En 1989, el Comité Económico y Social de las CCEE abogaba por la implantación de rentas mínimas de inserción (RMI). Por su lado, el Consejo de las CCEE también aprobó en 1992 una recomendación de establecer las RMI en todos los países comunitarios.

Las actuaciones europeas de lucha contra la pobreza y la exclusión social se han concretado en diferentes medidas y programas en los distintos países miembros. En el caso español el discurso europeo tiene su expresión en la puesta en marcha del denominado Programa para Situaciones de Necesidad, creado en 1987, para dar cauce a Pobreza (II). Posteriormente, en 1989 España incluyó también acciones de Pobreza (III) y con el cambio de década se introdujeron dos políticas que han supuesto el acceso a una protección mínima para una porción significativa de la población. En 1990 la Ley de Prestaciones no Contributivas de la Seguridad Social (26/1990) puso en marcha un sistema de pensiones para ancianos y minusválidos, en situaciones de necesidad probada, que no accedían a la Seguridad Social contributiva. En este mismo período, las CCAA elaboraron e implementaron los programas regionales de RMI dirigidos a la lucha contra la pobreza y la exclusión social. Se trata de la contribución más innovadora en el ámbito asistencial, en tanto que son programas de transferencia de rentas monetarias a familias de bajos ingresos, con el objetivo de la inserción social de los receptores (siguiendo las recomendaciones europeas y las líneas maestras del programa de RMI francés). El impulso de una buena parte de estos programas autonómicos vino de los propios gobiernos regionales como resultado de la acción combinada de políticos y técnicos. En algunos casos, el factor impulsor de estos programas fue la presión ejercida por los partidos de la oposición en los parlamentos regionales. Una tercera vía de impulso político se debió a la movilización de las secciones regionales de los principales sindicatos CCOO y UGT (debido a fractura de la negociación colectiva a escala central y apertura de la regional) y en algunas CCAA el apoyo de ONGs como Cáritas (Arriba, 2002). 
Los esquemas de RMI forman parte de la "última malla de seguridad" (safety net) en tanto que conjunto de derechos económicos, políticos y sociales que proveen un mínimo de recursos y medios para la subsistencia e inserción, a los ciudadanos que no pueden generarlos por sí mismos (Moreno, 2002:29). En España las RMI tienen un papel residual y subsidiario respecto de otros esquemas de protección de la Seguridad Social. Además recogen las consecuencias de los recortes, cambios e insuficiencias de los demás sistemas de bienestar, no sólo respecto a los ingresos, sino también en lo referido a vivienda, educación y salud. La implantación de los sistemas de rentas mínimas adoptados por las distintas CCAA a pesar de contar con elementos comunes en aspectos normativos y de concesión, han dado lugar a un complejo mosaico de denominaciones, contenidos, estrategias, recursos y resultados. Tras un primer período de implementación (1989-1995) y de pasar una "travesía del desierto", algunas CCAA han llevado a cabo reformas, reestructuraciones e incluso refundaciones de los programas, mientras otros han permanecido inalterados. Las iniciativas de interés han tenido lugar en algunos de los programas que ya estaban más desarrollados en los noventa. Así mismo resulta imposible la existencia de pautas de cambio comunes. Ayala (2000) ha estudiado estas relaciones poniéndolas en relación con los cambios en el empleo y los cambios demográficos de las propias políticas de rentas mínimas, sin conseguir establecer una relación significativa. Según Arriba (2006) son las variables institucionales las que inciden en los cambios de los programas. El origen de estos programas tuvo un carácter legitimador para los actores que mediaron en su puesta en marcha. Su desarrollo posterior muestra que se trata de programas vulnerables, sometidos a un elevado grado de discrecionalidad política.

En este período, los distintos programas de rentas mínimas parecen haber incrementado sus diferencias (Laparra, 2004), pero donde sí se detectan tendencias comunes es en la introducción de criterios de activación en los sistemas de asistencia social. Una gradual orientación hacia la activación de las políticas y programas a los que puede acceder la población en situación potencialmente activa: refuerzo de la orientación a la inserción en los programas autonómicos de renta mínima, introducción de criterios restrictivos de acceso a prestaciones y subsidios y transformaciones de políticas de mercado de trabajo (por ejemplo Renta Activa de Inserción). De esta forma, la relación entre trabajo y asistencia se ha convertido en el asunto central de la agenda política, transformando discursivamente la "última red de protección en un trampolín hacia el empleo" Las desigualdades sociales dejan de ser el problema central, para dejar paso a los desincentivos generados por las propias políticas o programas de prestaciones sociales. La cuestión central en los debates no es la pobreza sino la inclusión social (Aust y Arriba, 2006).

Marco interpretativo 5: Crecimiento, empleo y cohesión social (1997-2005)

Con la firma del Tratado de Ámsterdam de 1997, que incorpora el protocolo social, y la Cumbre de Luxemburgo del mismo año, que establecía una Estrategia Europea sobre el Empleo, la situación empieza a cambiar. El desarrollo más importante en la política social europea parte de la Cumbre de Lisboa en marzo del año 2000, donde los jefes de estado y de gobierno reconocen la importancia de to- 
\} LA IMPORTANCIA DE LAS IDEIAS EN EL CAMBIO DE PARADIGMA: (...) - ADELANTADO, J. e PÉREZ-GIMÉNEZ, R. \}

mar medidas concretas para fomentar la cohesión social en Europa. La llamada estrategia de Lisboa se basa en una profunda relación entre el triángulo: desarrollo económico, empleo y cohesión social y mostró gran optimismo al fijar como meta la erradicación de la pobreza en 2010. En la Cumbre de Lisboa también se crea el Método Abierto de Coordinación que fija el papel de la Comisión y de los estados miembros. A la primera, le otorga una función de estímulo, coordinación y potenciación de los intercambios y determina para los segundos la plena responsabilidad en la lucha contra la pobreza y la exclusión. Este método de coordinación queda asegurado por un alto comité, con la creación de un programa de Acción Comunitario, con los Planes Nacionales de Acción para la Inclusión, y la aplicación de los indicadores acordados en la Cumbre de Laeken.

Desde la aprobación en las cumbres de Lisboa y Niza del año 2000 del Método Abierto de Coordinación como instrumento de coordinación a escala europea de la política social desarrollada a escala nacional, el Reino de España, en el marco de la Estrategia Europea de Inclusión, ha elaborado I Plan Nacional de Acción para la Inclusión Social 2001-2003 (PNAIn-I) el (PNAIn-II) 2003-2005, el (PNAIn-III) 20052006 el (PNAIn-IV) 2006-2008 y el (PNAIn-V) 2008-2010, estructurados en torno a los objetivos comunes previamente establecidos en dicho marco. En cumplimiento de las líneas prioritarias establecidas por el Método Abierto de Coordinación, el PNAIn-II (2003-2005) avanzó en la coordinación con las correspondientes medidas del Plan Nacional de Acción para el Empleo en los siguientes aspectos: establecimiento de Planes Autonómicos de Inclusión social (PAls), incentivación de la participación de las personas afectadas, y en el planteamiento de una atención integral y coordinada a grupos y personas vulnerables, poniendo especial énfasis en la destinada a inmigrantes, infancia, mujer y minorías étnicas. Además del enfoque de género como un principio transversal. Respecto a los PNAIn-I y PNAIn-II, tras su presentación la Comisión realizó Informes conjuntos (2001) y (2004), en los que se constta la insistencia en el empleo y en las llamadas políticas activas en el conjunto de la protección social, en las políticas sectoriales (educación, salud, vivienda) y en algunos colectivos como la población infantil, los inmigrantes y las minorías étnicas. Los dos PNAIn reconocen que el sistema actual de descentralización territorial otorga amplias competencias a las CA en materias sustantivas para combatir la pobreza y la exclusión, y que los Ayuntamientos tienen funciones de gestión en ellas.

\section{Marco interpretativo 6: Crecimiento, empleo y gobernanza (2005-08)}

En noviembre del 2004, se presentó un informe encargado por la Comisión Europea a un grupo de "alto nivel" sobre los retos de la Unión, en el que el crecimiento y el empleo se establecían como las líneas de futuro de la revisión de la Agenda de Lisboa. Apenas unos meses después, en febrero de 2005 la Comisión europea decidió llevar a cabo un proceso de revisión de la estrategia de Lisboa dado los escasos progresos de la misma. Se publicó entonces una comunicación del Presidente de la Comisión titulada "Trabajando juntos por el crecimiento y el empleo-Relanzamiento de la estrategia de Lisboa" también conocida como "Informe Barroso". En ésta se marcan dos prioridades: el crecimiento económico y la creación de empleo, entendiendo que tanto la inclusión social como la cohesión social son 
consecuencias del crecimiento económico. La estrategia de Lisboa Renovada prevé no sólo el cumplimiento de una nueva agenda social europea (2005-2010) sino también el establecimiento de nuevos objetivos y nuevos indicadores. Si bien en 2000 los ejes eran crecimiento, empleo y cohesión social, la nueva agenda social desarrolla una estrategia alrededor del crecimiento y el empleo y, de forma complementaria, la gobernanza. En el Informe Conjunto sobre inclusión y protección social de 2006 es el primero que unifica el diagnóstico y las propuestas. Para la Comisión el fomento de la inclusión social se vincula a la consecución de las prioridades del empleo y en las Ilamadas políticas activas (en el conjunto de la protección social), en las políticas sectoriales (educación, salud, vivienda) y en algunos colectivos como la población infantil y las minorías étnicas.

Desde finales de los años 90 y principios del 2000, la mayoría de gobiernos autonómicos han promovido planes de inclusión social para luchar contra la pobreza y la exclusión social en sus territorios. En 14 de las 17 CCAA se ha aprobado al menos un plan de inclusión social. Las CCAA que tomaron la iniciativa fueron La Rioja, Castilla-La Mancha, Navarra y Canarias quienes aprobaron planes con anterioridad a la Cumbre de Lisboa de 2000 y el establecimiento del Método Abierto de Coordinación. La siguiente ola de planes se aprobó entre los años 2001-2004, coincidiendo con la presentación de los primeros PNAIn bajo el marco de la Estrategia Europea de Inclusión Social (Andalucía, Aragón, Castilla-León, Cataluña, Galicia, País Vasco, Madrid y Valencia). La última ola de planes se aprobó tras la revisión y relanzamiento de la Estrategia Europea de Lisboa (Asturias, Extremadura). En otras CCAA como Baleares, Cantabria o Murcia, no se ha ejecutado ningún plan. Sin embargo en todas las CCAA existen políticas impulsadas por los gobiernos autónomos cuyo objetivo es reducir la pobreza y la exclusión social.

Renes, Lorenzo y Chahin (2007: 80-81) presentan una matriz de aspectos temáticos y actores implicados en los PNIn. Forman parte temas como el empleo (en 11CA), salud (11), educación (11), atención a colectivos vulnerables (8), RMI (11), Servicios Sociales (6), vivienda (11), familia (5) y las nuevas tecnologías de la información (en 4 CA). Están implicadas en los PAls de Galicia y Madrid otras consejerías y corporaciones locales; en los de Castilla-La Mancha y Valencia, corporaciones locales y agentes sociales, y en los de Andalucía, Castilla-León, Cataluña y Extremadura, otras consejerías, corporaciones locales y agentes sociales. Nuevamente se constata la heterogeneidad de los planes en las diferentes CA tanto en lo que se refiere a aspectos temáticos como a actores implicados.

\section{Conclusiones}

Los seis marcos interpretativos que se han señalado respecto a las ideas y los discursos que contienen las leyes de SS y AS, describen un proceso de reforma en esta política y se ubican en un contexto caracterizado por la transición de las sociedades fordistas a las postfordistas, con la aparición de nuevos riesgos sociales y con el cambio del "modo de regulación" que ello conlleva. Más Europa y menos España, que reivindican los nuevos regionalismos; más mercado y menos Estado, que reivindican las nuevas ideas cognitivas (las que definen los problemas, las soluciones y los instrumentos), y una situación de austeridad fiscal permanente, cons- 
\} LA IMPORTANCIA DE LAS IDEIAS EN EL CAMBIO DE PARADIGMA: (...) - ADELANTADO, J. e PÉREZ-GIMÉNEZ, R. \}

tatan el cambio de paradigma. Dicho cambio procede de la conjugación de factores endógenos (estructura institucional doméstica) y exógenos (europeización), que habrían provocado la emergencia de nuevos actores (subnacionales y supranacionales, institucionales y extrainstitucionales, públicos y privados) portadores de nuevos intereses, ideas normativas (recetas para la acción política) y discursos.

En resumen, en la política de SS y AS se puede sistematizar en tres ciclos de desarrollo que muestran la transición de las aspiraciones a un modelo de Estado de Bienestar Keynesiano a otro de tipo Schumpeteriano alrededor del work-fare state. El primer ciclo se extiende desde la transición democrática hasta mediados de los noventa, el segundo entre mediados de los noventa y principios de los dos mil, y el tercero desde entonces hasta 2008.

Las primeras leyes de SS (1982-92) tenían por objetivo la universalización y responsabilidad pública y, aunque no culminaron su desarrollo, significaron una importante institucionalización de esta política (si bien siguieron operando en clave asistencialista, habida cuenta de la inexistencia de un derecho subjetivo). Los instrumentos para conseguirlos eran fundamentalmente públicos a través de una red institucional compuesta por consejerías, concejalías y centros de SS; y el discurso estaba fuertemente influenciado por el esquema cognitivo de técnicos y trabajadoras de SS. Las consecuencias del cambio de paradigma económico (del keynesianismo al monetarismo), especialmente el desempleo, y el acceso de España a la Comunidad Europea en 1986, facilitó que otra de las consecuencias, la pobreza, entrara en el escenario cognitivo. Entre (1989-95) se aprueban leyes de RMI en todas las CCAA; los objetivos de universalidad y responsabilidad pública que declaraban las leyes de SS se mantuvieron, igual que los instrumentos, pues se canalizaron a través de la red institucional y también eran asistencialistas. Pero los actores se modificaron: las recomendaciones de las instituciones europeas, los sindicatos, Cáritas, y los partidos opositores en los parlamentos regionales fueron los principales impulsores. El discurso que manejan los actores gira alrededor del derecho a la inserción en un doble sentido, procurar subsistencia e inserción socio-laboral.

El segundo ciclo de la política de SS y AS es de transición de un paradigma a otro y se extiende desde mediados de los noventa a principios de los dos mil. En este período se produce una adaptación gradual con renovación parcial de las instituciones y redirección de los principios path departure. Así como un cambio de instrumentos (segundo orden de cambio) y una reacción adaptativa a los cambios inducidos por la UE en forma de "absorción". En las reformas de las leyes autonómicas de SS se observa un tránsito desde los objetivos de universalismo y responsabilidad pública hacia los de privatización y altruismo, mientras que las RMI sufren una "travesía del desierto". Los instrumentos dejan de ser exclusivamente públicos, pues se reconoce la presencia de la iniciativa privada lucrativa y se delegan funciones de prestación de servicios en el Tercer Sector. La emergencia de nuevos actores se explica por la potencia sustantiva y comunicativa para promover un cambio de marco cognitivo que se puede resumir en menos estado, más mercado y más voluntariado. Las críticas a los monopolios de los servicios públicos del estado del bienestar, dio paso a la sociedad del bienestar con nuevos imaginarios sobre lo que deben ser y cómo han de actuar las administraciones públicas. Contribuyó a 
ello, o se aprovechó, la llamada estrategia de Lisboa basada en el crecimiento económico, del empleo y como consecuencia de la cohesión social. Las políticas asistenciales como las RMI (adscritas a la Estretegia Europea de Inclusión social) se vinculan con las políticas laborales de empleo; a su vez, la laxitud en el cumplimiento de objetivos que posibilita el Método Abierto de Coordinación, ha hecho posible la producción de Planes Nacionales para la Inclusión social que otorgan una enorme discrecionalidad a los estados miembros y a las instituciones subnacionales.

En el último ciclo, desde principios de los dos mil hasta 2008, se consolida el cambio de paradigma. Las nuevas leyes de SS avanzan en un doble sentido, subjetivación de derechos y aportación económica de los beneficiarios, a la vez que se consolida la presencia de la iniciativa privada mercantil y altruista. Las RMI avanzan en la incorporación diferenciada del derecho a la prestación económica y de actuaciones dirigidas a la integración laboral, mientras se profundiza el vínculo con las políticas de empleo, especialmente a escala local. A lo largo de este período han cesado las herencias universalistas y de responsabilidad pública path cessation, han cambiado los instrumentos y los objetivos, y por tanto los discursos ("tercer orden de cambio") y ha cesado la resistencia a los cambios inducidos por la UE ("trasformación de la política"). Por una parte, en la política de SS y AS se observa la profundización de los derechos de ciudadanía y, por otra, un crecimiento de condicionalidades para acceder a la asistencia (especialmente entre los pobres capaces). La subjetivación de derechos es importante porque supone la posibilidad de conseguir la vieja aspiración de los Estados del Bienestar de blindar un mínimo de subsistencia garantizado por ley. Sin embargo, tanto el sistema de financiación de los nuevos derechos, que se realiza mediante un copago del beneficiario en función de su renta y patrimonio (incluida la vivienda) y no mediante impuestos generales, como la opción por los cheques de distinto tipo (por servicio, por bebé...) en lugar de promover servicios públicos como residencias o guarderías, dan cuenta de la consolidación del cambio. En este período la UE ha revisado la estrategia de Lisboa, pero a pesar de la persistencia de la pobreza y la exclusión, sus orientaciones se dirigen más bien a la fusión de la Estrategia Europea de Empleo con la Estrategia Europea de Inclusión social, mediante en concepto de "activación", esto es, hacer que el trabajar sea rentable (making work pay). 


\section{Bibliografía}

Alemán, C. y García Serrano, M. (2005) Servicios Sociales Sectoriales. Madrid: Editorial universitaria Ramón Areces.

Arriba, Ana (2002) "Procesos de implantación de políticas de Rentas Mínimas de Inserción en España" en Luis Moreno (ed.): Pobreza y exclusión: la "Malla de seguridad" en España. Madrid: CSIC. pp. 175-220

Arriba, Ana (2006): "Las reformas de la protección social frente a la pobreza: Asistencia social y garantía de mínimos" en G. Rodríguez Cabrero, A. Arriba, Vicente Marbán y O. Salido: Actores Sociales y reformas del bienestar. Madrid. CSIC-UPC. pp. 115-143.

Aust, Andreas; Arriba, Ana (2006) "Towards Activation? Social Assistance Reforms and Discourses" en Peter Taylor-Gooby (ed.): Ideas and Welfare State Reform in Western Europe. New York: Palgrave-Macmillan. pp. 100-123

Börzel, Tanja A (2002): "Pace-Setting, Foot-Dragging and Fence-Sitting: Member State Responses to Europeanization". Journal of Common Market Studies. Vol. 40 Number 2. pp: 193-214.

Bulmer, Simon y Radaelli, Claudio M (2003) "The Europeanization of National Policy" en Simon Bulmer, Simon y Christian Lequesne (eds.): The Member States of the European Union. Oxford: Oxford University Press.

Casado, Demetrio (2007) "La rama de los servicios sociales" en Demetrio Casado y Fernando Fantova (coords.): Perfeccionamiento de los servicios sociales en España. Informe con ocasión de la Ley sobre autonomía y dependencia. Madrid: Cáritas Española Editores y Fundación FOESSA. pp. 101-145

Castel, Robert (2002) "Du travail social à la gestion du non-travail" en Claudine Leleux (ed.): L'assistant social entre aide et contrôle social. IESSID: Bruxelles. pp.167192

Ebbinghaus, Bernhard (2005) "Can Path Dependence Explain Institutional Change? Two Approaches Applied to Welfare State Reform". Max Planck Institute for the Study of Societies. Discussion Paper 05/2

Featherstone, Kevin (2003): "Introduction: In the Name of "Europe" en Kevin Featherstone y Claudio M. Radaelli (eds): The Politics of Europeanization. Oxford: Oxford University Press. pp. 3-26

Guillén, Encarna y Vilà, Antoni (2007) "Los cambios legislativos recientes en materia de servicios sociales" en Demetrio Casado y Fernando Fantova (coords): Perfeccionamiento de los servicios sociales en España. Informe con ocasión de la Ley sobre autonomía y dependencia. Madrid: Caritas Española Editores y Fundación FOESSA. pp. 147-175

Hall, Peter (1993) "Policy Paradigms, Social Learning, and the State: The Case of Economic Policymaking in Britan". Comparative Politics, Vol. 25, №. 3 (Apr., 1993), pp. 275-296

Hooghe, Liesbet y Marks, Gary (2001) Miultilevel Governance and European Integration. Langham: Rowman and Littlefied 
Jessop, Bob (2004) "Critical Semiotic Analysis and Cultural Political Economy" Critical Discourse Studies Vol. 1 (issue 2) october, pages 159-174

Jessop, Bob (2007): State Power: A Strategic-Relational Approach. London: Polity

Laparra, Miguel (2004) "La travesía del desierto de las rentas mínimas en España. Documentación Social, no 135: 57-76

Loughlin, John (2007): "Reconfiguring the State: Trends in Territorial Governance in European States", Regional and Federal Studies. Vol 17, Number 4. pp. 385-403

Mahoney, James (2000) "Path Dependence in Historical Sociology". Theory and Society, Vol. 29, No. 4. (Aug., 2000), pp. 507-548

Moreno, Luis (2002) "Estados del bienestar y "Mallas de seguridad". En Luis Moreno (ed.): Pobreza y exclusión: la "Malla de seguridad" en España. Madrid. CSIC. Págs. 17-50

Olsen, Johan P. (2002): "The Many Faces of Europeanization". Journal of Common Market Studies. Vol 40, Number 5. pp. 921-52.

Pierson, Paul (2001): "Coping with Permanent Austerity: Welfare State Restructuring in Affluent Democracies" en P. Pierson (ed.): The New Politics of the Welfare State. Oxford. Oxford University Press. Págs. 410-456.

Powell, Charles T (2007) "La larga marcha hacia Europa: España y la Comunidad Europea (1957-1986). En Francesc Morata y Gemma Mateo (eds.): España en EuropaEuropa en España (1986-2006). Barcelona: Fundación CIDOB. pp. 41-67

Radaelli, Claudio M (2003): "The Europeanization of Public Policy" en Kevin Featherstone y Claudio M. Radaelli (eds); The Politics of Europeanization. Oxford: Oxford University Press. pp. 27-56.

Renes, Víctor, Lorenzo, Francisco y Chahin, Alia (2007) "Poniendo en práctica la Estrategia Europea para la Inclusión Social. Del plano europeo al plano local". Fundación Luis Vives. Cuaderno europeo 4.

Risse, T; Cowles, M.G; and Caporaso (2001) "Europeanization and Domestic Change: Introduction" en M.G. Cowles, J. Caporado and T. Risse (eds.): Transforming Europe. Europeanization and domesctic change. Ithaca: Cornell University Press. pp. 1-20

Roldán García, Elena y García Giráldez, Teresa (2006): Políticas de Servicios Sociales. Madrid: Síntesis.

Schmidt, Vivien A. (2008) "Discursive Institutionalism: The Explanatory Power of Ideas and Discourse". Annual Review of Political Science, 11:303-26

Snow, David y Benford, Robert (1992) "Master Frames and Cycles of Protest" en Aldon Morris y Carol Mueller (eds.): Frontiers in Social Movement Theory. New Haven: Yale University Press, pp. 133-155.

Vink, Maarte P. y Graziano, Paolo (2007) "Challenges of a New Research Agenda" en Paolo Graziano y Maarten P. Vink (eds.): Europeanization. New Research Agenda. New Yok: Palgrave-macmillan. pp. 3-20.

Recebido em 28 de setembro de 2010.

Aceito para publicação em 5 de novembro de 2010. 